\title{
Transformation operators and their applications for modeling in two-layer media
}

\author{
O.E. Yaremko *, N.N. Yaremko \\ Penza State University, Penza, Russia \\ *Corresponding author E-mail: yaremki@mail.ru
}

Copyright (C)2014 O.E. Yaremko and N.N. Yaremko . This is an open access article distributed under the Creative Commons Attribution License, which permits unrestricted use, distribution, and reproduction in any medium, provided the original work is properly cited.

\begin{abstract}
We present a transformation operators method which allows us to interpret piecewise-homogeneous physical processes as a perturbing of a homogeneous ones. The analytical description of mathematical models of thermal conductivity and wave processes for piecewise homogeneous media with flat symmetry is obtained by the developed in this paper transformation operators method.
\end{abstract}

Keywords: Poisson formula, Wave equation, Heat equation, Dirichlet problem, Laplace equation.

\section{1. introduction}

The important mathematical models describing physical fields in multilayered piecewise homogeneous media, lead to an initial boundary value problems for partial differential equations[6]. The coefficients of the equations are continuous for homogeneous models and the coefficients of the equation are piecewise constant for piecewise-homogeneous models. Transformation operators allow to interpret piecewise-homogeneous physical fields as a perturbing homogeneous fields. The authors offer to use transformation operators method $[8,12]$ for the study of mathematical models in piecewise homogeneous media. Classical transformation operators such as Weierstrass, Poisson, Sonin are treated as operators of perturbing. Theory of the classical transformation operators was developed by Marchenko V.A. [1], Kipriyanov I.S. [2], Samko, S.G., Kilbas, A.A., Marichev, O.I. [3]. The authors suggest to consider the transformation operator as a deformation of the mathematical homogeneous model into a piecewise-homogeneous model. In this work transformation operators are constructed, studied and used for problems' solving on the composite real line.The generalized expressions of d'Alembert and Poisson integrals [4], [5] over the composite real line are obtained. The analytical description of mathematical models of thermal conductivity $[5,7]$ and wave processes for piecewise homogeneous media with flat symmetry is received. The Dirichlet problem for Laplace equation $[5,6]$ on the composite real upper half-plane is solved.

\section{Transformation operators for a composite real line}

Theorem 2.1 If the function

$$
\widetilde{f}(x)=\theta(l-x) \widetilde{f}_{1}(x)+\theta(x-l) \widetilde{f}_{2}(x)
$$


is determined on a composite real line $I_{1}=(-\infty, l) \cup(l, \infty)$ and satisfies the following relations at the point $x=l$

$$
\tilde{f}_{1}(l-)=\tilde{f}_{2}(l+), \tilde{k} \tilde{f}_{1}^{\prime}(l-)=\tilde{f}_{2}^{\prime}(l+)
$$

then the function

$$
f(x)=\theta(l-x) f_{1}(x)+\theta(x-l) f_{2}(x),
$$

$f_{1}(x)=\frac{\frac{a_{1}}{a_{2}}+k}{\frac{\widetilde{a}_{1}}{\widetilde{a}_{2}}+\widetilde{k}} \cdot \frac{\widetilde{k}}{k} \widetilde{f}_{1}\left(\frac{\widetilde{a}_{1}}{a_{1}} x\right)-\frac{\widetilde{k} \frac{a_{1}}{a_{2}}-k \frac{\widetilde{a}_{1}}{\widetilde{a}_{2}}}{\left(\frac{\widetilde{a}_{1}}{\widetilde{a} 2}+\widetilde{k}\right) k} \widetilde{f}_{2}\left(-\frac{\widetilde{a}_{2}}{a_{1}} x\right), x<0 ;$

$f_{2}(x)=\widetilde{f}_{2}\left(\frac{\widetilde{a}_{2}}{a_{2}} x\right), 0<x$

is determined on a composite real line $I_{1}=(-\infty, l) \cup(l, \infty)$ and satisfies the following relations at the point $x=l$

$f_{1}(l)=f_{2}(l), k f_{1}^{\prime}(l)=f_{2}^{\prime}(l)$

Proof. The following equation is true

$f_{1}(0)=\frac{\frac{a_{1}}{a_{2}}+k}{\widetilde{a}_{1}+\widetilde{k}} \cdot \frac{\widetilde{k}}{\widetilde{a}_{2}} \widetilde{f}_{1}(0)-\frac{\widetilde{k} \frac{a_{1}}{a_{2}}-k \frac{\widetilde{a}_{1}}{\widetilde{a}_{2}}}{\left(\frac{\widetilde{a}_{1}}{\widetilde{a} 2}+\widetilde{k}\right) k} \widetilde{f}_{2}(0)=\widetilde{f}_{2}(0)=f_{2}(0)$.

Similarly, the following equality is true

$$
\begin{aligned}
& f_{1}^{\prime}(0)=\frac{\frac{a_{1}}{a_{2}}+k}{\widetilde{a}_{1}+\widetilde{a}} \cdot \frac{\widetilde{k}}{\widetilde{a}_{2}} \frac{\widetilde{a}_{1}}{a_{1}} \widetilde{f}_{1}^{\prime}(0)+\frac{\widetilde{k} \frac{a_{1}}{a_{2}}-k \frac{\tilde{a}_{1}}{\widetilde{a}_{2}}}{\left(\frac{\widetilde{a}_{1}}{\widetilde{a} 2}+\widetilde{k}\right) k} \frac{\widetilde{a}_{2}}{a_{1}} \widetilde{f}_{2}^{\prime}(0)=\frac{\frac{a_{1}}{a_{2}}+k}{\frac{\widetilde{a}_{1}}{\widetilde{a}_{2}}+\widetilde{k}} \cdot \frac{1}{k} \frac{\widetilde{a}_{1}}{a_{1}} \widetilde{f}_{2}^{\prime}(0)+ \\
& +\frac{\widetilde{k} \frac{a_{1}}{a_{2}}-k \frac{\widetilde{a}_{1}}{\widetilde{a}_{2}}}{\left(\frac{\widetilde{a}_{1}}{\widetilde{a} 2}+\widetilde{k}\right) k} \frac{\widetilde{a}_{2}}{a_{1}} \widetilde{f}_{2}^{\prime}(0)=\frac{\widetilde{a}_{2}}{a_{2} k} \widetilde{f}_{2}^{\prime}(0)=\frac{f_{2}^{\prime}(0)}{k} .
\end{aligned}
$$

Definition $2.2,[10]$. The transforms $J^{-1}: \widetilde{f}(x) \rightarrow f(x), \quad J: f(x) \rightarrow \widetilde{f}(x)$ are called the direct and inverse transformation operators, respectively.

\section{Wave equation on the composite real line}

Let $u(t, x)$ denote the vertical displacement of a string from the $x$-axis at position $x$ in time $t$. We assume that the string possesses is undergoing small amplitude transverse vibrations so that satisfies the wave equation [5]

$$
u_{t t}-a^{2} u_{x x}=0
$$

for all $-\infty<x<\infty$ and $t>0$. We also assume that we know the position and speed of the string in time $t=0$. We are given an initial displacement $u(x, 0)=f(x)$ and initial velocity $u_{t}(x, 0)=0$.

Consider the initial value problem

$$
\left\{\begin{array}{c}
u_{t t}-a^{2} u_{x x}=0, \quad t>0, x \in R \\
u(0, x)=f(x), \quad x \in R \\
u^{\prime}{ }_{t}(0, x)=f(x), \quad x \in R
\end{array}\right.
$$

The solution of initial value problem is given by the d'Alembert formula:

$u(t, x)=\frac{f(x-a t)+f(x+a t)}{2}$.

Let us consider two initial boundary value problems. 
First:

$$
\left\{\begin{array}{cc}
u_{i t t}-a_{i}^{2} u_{i x x}=0, & t>0, x \in\left(l_{i-1}, l_{i}\right) \\
u_{i}(0, x)=f_{i}(x), & x \in\left(l_{i-1}, l_{i}\right), i=1,2 \\
u_{1}(t, 0)=u_{2}(t, 0), & t>0 \\
k u_{1}^{\prime}(t, 0)=u_{2}^{\prime}(t, 0), & t>0
\end{array}\right.
$$

Second

$$
\left\{\begin{array}{cc}
\tilde{u}_{i t t}-\tilde{a}_{i}^{2} \tilde{u}_{i x x}=0, & t>0, x \in\left(l_{i-1}, l_{i}\right) \\
\tilde{u}_{i}(0, x)=\widetilde{f}_{i}(x), & x \in\left(l_{i-1}, l_{i}\right), i=1,2 \\
\tilde{u}_{1}(t, 0)=\tilde{u}_{2}(t, 0), & t>0 \\
\tilde{k} \tilde{u}_{1}^{\prime}(t, 0)=\tilde{u}_{2}^{\prime}(t, 0), & t>0
\end{array}\right.
$$

where $l_{0}=-\infty, l_{1}=0, l_{2}=\infty$

Theorem 3.1 If the function

$$
\widetilde{u}(t, x)=\theta(l-x) \widetilde{u}_{1}(t, x)+\theta(x-l) \widetilde{u}_{2}(t, x)
$$

is a solution of the initial value problem (4) and an initial displacement is defined by

$\widetilde{f}_{1}(x)=\frac{\frac{\widetilde{a}_{1}}{\tilde{a}_{2}}+\widetilde{k}}{\frac{a_{1}}{a_{2}}+k} \cdot \frac{k}{\widetilde{k}} f_{1}\left(\frac{a_{1}}{\widetilde{a}_{1}} x\right)-\frac{k \frac{\tilde{a}_{1}}{\tilde{a}_{2}}-\widetilde{k} \frac{a_{1}}{a_{2}}}{\left(\frac{a_{1}}{a 2}+k\right) \tilde{k}} f_{2}\left(-\frac{a_{2}}{\widetilde{a}_{1}} x\right), x<0 ;$

$\widetilde{f}_{2}(x)=f_{2}\left(\frac{a_{2}}{\widetilde{a}_{2}} x\right), 0<x$

then the function

$$
u(t, x)=\theta(l-x) u_{1}(t, x)+\theta(x-l) u_{2}(t, x)
$$

where

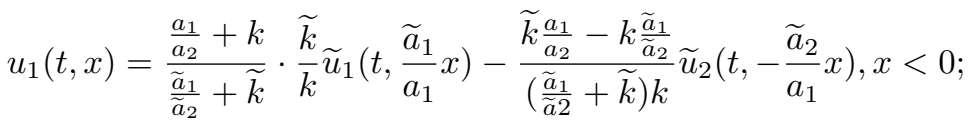

$u_{2}(t, x)=\widetilde{u}_{2}\left(t, \frac{\widetilde{a}_{2}}{a_{2}} x\right), 0<x$

is a solution of the initial value problem (3).

Proof. For operator

$$
B=\theta(l-x) a_{1}^{2} \partial_{x x}+\theta(x-l) a_{2}^{2} \partial_{x x}
$$

the following identity $\widetilde{B u}=\tilde{B} \tilde{u}$ holds true,

where

$$
\widetilde{B}=\theta(l-x) \tilde{a}_{1}^{2} \partial_{x x}+\theta(x-l) \tilde{a}_{2}^{2} \partial_{x x} .
$$

Applying Theorem 1, we have complete the proof.

Corollary 3.2 If in (5)-(6) we put $\tilde{a}_{1}=1, \tilde{a}_{2}=1, \tilde{k}=1$, then the solution of problem (5)-(6) has the form:

$u_{1}(t, x)=\frac{\frac{a_{1}}{a_{2}}+k}{2 k} \widetilde{u}_{1}\left(t, \frac{x}{a_{1}}\right)-\frac{\frac{a_{1}}{a_{2}}-k}{2 k} \widetilde{u}_{2}\left(t,-\frac{x}{a_{1}}\right), x<0 ;$

$u_{2}(t, x)=\widetilde{u}_{2}\left(t, \frac{x}{a_{2}}\right), 0<x$

Now we can prove an analogue of the d'Alembert formula for the initial boundary value problem (3) . 
Corollary 3.3 The solution of the initial boundary value problem (3) is given by the similar d'Alembert formula:

$u_{1}(t, x)=\frac{\frac{a_{1}}{a_{2}}+k}{4 k}\left(\tilde{f}\left(\frac{x}{a_{1}}-t\right)+\widetilde{f}\left(\frac{x}{a_{1}}+t\right)\right)-$

$-\frac{\frac{a_{1}}{a_{2}}-k}{4 k}\left(\tilde{f}\left(-\frac{x}{a_{1}}-t\right)+\tilde{f}\left(-\frac{x}{a_{1}}+t\right)\right), x<0$,

$u_{2}(t, x)=\frac{\tilde{f}\left(\frac{x}{a_{2}}-t\right)+\tilde{f}\left(\frac{x}{a_{2}}+t\right)}{2}, 0<x$

Remarks. We divide the half-plane $t>0,-\infty<x<\infty$ into 16 parts by using straight lines $x=a_{1} t, x=$ $a_{2} t, x=-a_{1} t, x=-a_{2} t$. In each of the 16 corners the analogue of the d'Alembert formulae in terms of the initial displacements $f_{1}(x), f_{2}(x)$ can be written. For example, in domain

$$
x+a_{1} t>0, x+a_{2} t>0, x-a_{1} t>0, x-a_{1} t>0
$$

analogue of the d'Alembert formula takes the form

$$
\begin{aligned}
& u_{1}(t, x)=\frac{\frac{a_{1}}{a_{2}}}{\frac{a_{1}}{a_{2}}+k}\left(f_{2}\left(\frac{a_{2}}{a_{1}} x-a_{2} t\right)+f_{2}\left(\frac{a_{2}}{a_{1}} x+t\right)\right)- \\
& -\frac{\frac{a_{1}}{a_{2}}-k}{\frac{a_{1}}{a_{2}}+k}\left(f_{1}\left(-x-a_{1} t\right)+f_{1}\left(-x+a_{1} t\right)\right), x<0, \\
& u_{2}(t, x)=\frac{f_{2}\left(x-a_{2} t\right)+f_{2}\left(x+a_{2} t\right)}{2}, 0<x .
\end{aligned}
$$

\section{Heat equation on composite real line}

In the special case of heat propagation in an isotropic and homogeneous medium in the first-dimensional space, heat conduction equation is [4]

$$
u_{t}^{\prime}=k u_{x x}^{\prime \prime}
$$

where $u=u(t, x)$ is a temperature as a function of time and space; $u_{t}^{\prime}$ is the rate of change of temperature at the point $x ; u_{x x}$ is the second- order partial derivatives (thermal conduction) of temperature, $k$ is a materialspecific quantity depending on the thermal conductivity, the density and the heat capacity. The heat equation is a consequence of Fourier's law of cooling.

If the medium is not the whole space, in order to solve uniquely heat equation we also need to specify boundary conditions for $u$. With an eye to determine uniqueness of solution in the whole space it is necessary to assume an exponential bound on the growth of solution, this assumption is consistent with observed experiments. Let $u(x ; t)$ is a temperature as a function of time and space. We assume that $u(x ; t)$ satisfies the heat equation

$$
u_{t}-a^{2} u_{x x}=0
$$

for all $-\infty<x<\infty$ and $t>0$. We also assume that we know the initial temperature of the rod in time $t=0$. We are given an initial distribution of temperature field $u(x, 0)=f(x)$

Let us consider the initial value problem

$$
\left\{\begin{array}{c}
u_{t}-a^{2} u_{x x}=0, \quad t>0, x \in R \\
u(0, x)=f(x), \quad x \in R
\end{array}\right.
$$

This is usually called the Cauchy problem for the heat equation in first- dimensional space. If we consider this equation on the whole real line, then we do not need to worry about boundary conditions and the solution is given by Poisson 's formula:

$u(t, x)=\frac{1}{2 \sqrt{\pi t}} \int_{-\infty}^{\infty} e^{-\frac{(x-\xi)^{2}}{4 t}} f(\xi) d \xi$ 
Consider two initial boundary value problems over the composite real line.

First:

$$
\left\{\begin{array}{cc}
u_{i t}-a_{i}^{2} u_{i x x}=0, & t>0, x \in\left(l_{i-1}, l_{i}\right) \\
u_{i}(0, x)=f_{i}(x), & x \in\left(l_{i-1}, l_{i}\right), i=1,2 ; \\
u_{1}(t, 0)=u_{2}(t, 0), & t>0 \\
k u_{1}^{\prime}(t, 0)=u_{2}^{\prime}(t, 0), & t>0
\end{array}\right.
$$

Second:

$$
\left\{\begin{array}{cc}
\tilde{u}_{i t}-\tilde{a}_{i}^{2} \tilde{u}_{i x x}=0, & t>0, x \in\left(l_{i-1}, l_{i}\right) \\
\tilde{u}_{i}(0, x)=\widetilde{f}_{i}(x), & x \in\left(l_{i-1}, l_{i}\right), i=1,2 ; \\
\tilde{u}_{1}(t, 0)=\tilde{u}_{2}(t, 0), & t>0 \\
\tilde{k} \tilde{u}_{1}^{\prime}(t, 0)=\tilde{u}_{2}^{\prime}(t, 0), & t>0
\end{array}\right.
$$

where $l_{0}=-\infty, l_{1}=0, l_{2}=\infty$

Theorem 4.1 If the function

$$
\widetilde{u}(t, x)=\theta(l-x) \widetilde{u}_{1}(t, x)+\theta(x-l) \widetilde{u}_{2}(t, x)
$$

is a solution of the initial value problem (8) and an initial displacement is defined by formulas

$\widetilde{f}_{1}(x)=\frac{\frac{\tilde{a}_{1}}{\bar{a}_{2}}+\widetilde{k}}{\frac{a_{1}}{a_{2}}+k} \cdot \frac{k}{\widetilde{k}} f_{1}\left(\frac{a_{1}}{\widetilde{a}_{1}} x\right)-\frac{k \frac{\widetilde{a}_{1}}{\tilde{a}_{2}}-\widetilde{k} \frac{a_{1}}{a_{2}}}{\left(\frac{a_{1}}{a 2}+k\right) \tilde{k}} f_{2}\left(-\frac{a_{2}}{\widetilde{a}_{1}} x\right), x<0 ;$

$\widetilde{f}_{2}(x)=f_{2}\left(\frac{a_{2}}{\widetilde{a}_{2}} x\right), 0<x$

then the function

$$
u(t, x)=\theta(l-x) u_{1}(t, x)+\theta(x-l) u_{2}(t, x)
$$

where

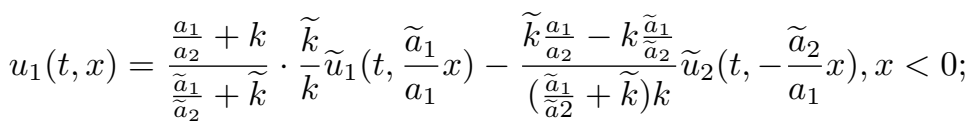

$u_{2}(t, x)=\widetilde{u}_{2}\left(t, \frac{\widetilde{a}_{2}}{a_{2}} x\right), 0<x$

is a solution of the initial value problem (7).

Proof. For operator

$$
B=\theta(l-x) a_{1}^{2} \partial_{x x}+\theta(x-l) a_{2}^{2} \partial_{x x}
$$

the following identity $\widetilde{B u}=\tilde{B} \tilde{u}$ is true,

where

$$
\widetilde{B}=\theta(l-x) \tilde{a}_{1}^{2} \partial_{x x}+\theta(x-l) \tilde{a}_{2}^{2} \partial_{x x} .
$$

Applying Theorem 1, we have complete the proof.

Corollary 4.2 If in (9)-(10) we put $\tilde{a}_{1}=1, \tilde{a}_{2}=1, \tilde{k}=1$, then the solution of problem (9)-(10) has the form:

$u_{1}(t, x)=\frac{\frac{a_{1}}{a_{2}}+k}{2 k} \widetilde{u}_{1}\left(t, \frac{x}{a_{1}}\right)-\frac{\frac{a_{1}}{a_{2}}-k}{2 k} \widetilde{u}_{2}\left(t,-\frac{x}{a_{1}}\right), x<0 ;$

$u_{2}(t, x)=\widetilde{u}_{2}\left(t, \frac{x}{a_{2}}\right), 0<x$

Corollary 4.3 The solution of the initial boundary value problem (7) is given by similar Poisson's formula: 


$$
\begin{aligned}
& u_{2}(t, x)=\frac{1}{2 \sqrt{\pi t}} \frac{2 k}{\frac{a_{1}}{a_{2}}+k} \int_{-\infty}^{0} e^{-\frac{\left(\frac{x}{a_{2}}-\xi\right)^{2}}{4 t}} f_{1}\left(a_{1} \xi\right) d \xi+ \\
& +\frac{1}{2 \sqrt{\pi t}} \int_{0}^{\infty}\left(\frac{\frac{a_{1}}{a_{2}}-k}{\frac{a_{1}}{a_{2}}+k} e^{-\frac{\left(\frac{x}{a_{2}}+\xi\right)^{2}}{4 t}}+e^{-\frac{\left(\frac{x}{a_{2}}-\xi\right)^{2}}{4 t}}\right) f_{2}\left(a_{2} \xi\right) d \xi, x>0 \\
& u_{1}(t, x)=\frac{1}{2 \sqrt{\pi t}} \int_{-\infty}^{0}\left(\frac{k-\frac{a_{1}}{a_{2}}}{\frac{a_{1}}{a_{2}}+k} e^{-\frac{\left(\frac{x}{a_{1}}+\xi\right)^{2}}{4 t}}+e^{-\frac{\left(\frac{x}{a_{1}}-\xi\right)^{2}}{4 t}}\right) f_{1}\left(a_{1} \xi\right) d \xi \\
& +\frac{1}{2 \sqrt{\pi t}} \int_{0}^{\infty} \frac{2 \frac{a_{1}}{a_{2}}}{\frac{a_{1}}{a_{2}}+k} e^{-\frac{\left(\frac{x}{a_{1}}-\xi\right)^{2}}{4 t}} f_{2}\left(a_{2} \xi\right) d \xi, \quad x<0
\end{aligned}
$$

\section{The Dirichlet problem for the Laplace equation}

Let $\hat{u}=\hat{u}(x, y)$ be harmonic on the upper half-plane $H=\{(x, y): 0<y, x \in R\}$, continuous on $H=\{(x, y): 0 \leq y, x \in R\}$ and satisfies the conditions

$\widehat{u}=o\left(\sqrt{x^{2}+y^{2}}\right)$,

$\int_{-\infty}^{\infty} \frac{\widehat{f}(\xi)}{1+\xi^{2}} d \xi<\infty$

then the function

$\widetilde{u}(x, y)=\frac{y}{\pi} \int_{-\infty}^{\infty} \frac{\widetilde{f}(\xi)}{(x-\xi)^{2}+y^{2}} d \xi$

is harmonic in the upper half plane $y>0$ and has the boundary values $\widetilde{u}(0, x)=\widetilde{f}(x)$ wherever $\widetilde{f}(x)$ is continuous.

The last formula is called Poisson integral formula [7]. Consider two initial boundary value problems over the composite real line.

First:

$$
\left\{\begin{array}{cc}
u_{i y y}+a_{i}^{2} u_{i x x}=0, & y>0, x \in\left(l_{i-1}, l_{i}\right) \\
u_{i}(0, x)=f_{i}(x), & x \in\left(l_{i-1}, l_{i}\right), i=1,2 \\
u_{1}(y, 0)=u_{2}(y, 0), & y>0 \\
k u_{1}^{\prime}(y, 0)=u_{2}^{\prime}(y, 0), & y>0
\end{array}\right.
$$

Second:

$$
\left\{\begin{array}{cc}
\tilde{u}_{i y y}+\tilde{a}_{i}^{2} \tilde{u}_{i x x}=0, & y>0, x \in\left(l_{i-1}, l_{i}\right) \\
\tilde{u}_{i}(0, x)=\widetilde{f}_{i}(x), & x \in\left(l_{i-1}, l_{i}\right), i=1,2 ; \\
\tilde{u}_{1}(y, 0)=\tilde{u}_{2}(y, 0), & y>0 \\
\tilde{k} \tilde{u}_{1}^{\prime}(y, 0)=\tilde{u}_{2}^{\prime}(y, 0), & y>0
\end{array}\right.
$$

where $l_{0}=-\infty, l_{1}=0, l_{2}=\infty$

Theorem 5.1 If the function

$$
\widetilde{u}(y, x)=\theta(l-x) \widetilde{u}_{1}(y, x)+\theta(x-l) \widetilde{u}_{2}(y, x)
$$

is a solution of the initial value problem (12) and an initial displacement is defined by formulas

$\tilde{f}_{1}(x)=\frac{\frac{\widetilde{a}_{1}}{\tilde{a}_{2}}+\widetilde{k}}{\frac{a_{1}}{a_{2}}+k} \cdot \frac{k}{\widetilde{k}} f_{1}\left(\frac{a_{1}}{\widetilde{a}_{1}} x\right)-\frac{k \frac{\widetilde{a}_{1}}{\tilde{a}_{2}}-\widetilde{k} \frac{a_{1}}{a_{2}}}{\left(\frac{a_{1}}{a 2}+k\right) \tilde{k}} f_{2}\left(-\frac{a_{2}}{\widetilde{a}_{1}} x\right), x<0 ;$

$\widetilde{f}_{2}(x)=f_{2}\left(\frac{a_{2}}{\widetilde{a}_{2}} x\right), 0<x$

then the function

$$
u(y, x)=\theta(l-x) u_{1}(y, x)+\theta(x-l) u_{2}(y, x)
$$


defined by formulas

$u_{1}(y, x)=\frac{\frac{a_{1}}{a_{2}}+k}{\widetilde{a}_{1}+\widetilde{k}} \cdot \frac{\widetilde{k}}{\widetilde{a}_{2}} \widetilde{u}_{1}\left(y, \frac{\widetilde{a}_{1}}{a_{1}} x\right)-\frac{\widetilde{k} \frac{a_{1}}{a_{2}}-k \frac{\widetilde{a}_{1}}{\tilde{a}_{2}}}{\left(\frac{\widetilde{a}_{1}}{\widetilde{a} 2}+\widetilde{k}\right) k} \widetilde{u}_{2}\left(y,-\frac{\widetilde{a}_{2}}{a_{1}} x\right), x<0 ;$

$u_{2}(y, x)=\widetilde{u}_{2}\left(y, \frac{\widetilde{a}_{2}}{a_{2}} x\right), 0<x$

is a solution of the initial value problem (11).

Proof. For operator

$$
B=\theta(l-x) a_{1}^{2} \partial_{x x}+\theta(x-l) a_{2}^{2} \partial_{x x}
$$

the following identity $\widetilde{B u}=\tilde{B} \tilde{u}$ is true,

where

$$
\widetilde{B}=\theta(l-x) \tilde{a}_{1}^{2} \partial_{x x}+\theta(x-l) \tilde{a}_{2}^{2} \partial_{x x} .
$$

Applying Theorem 1, we have complete the proof.

Corollary 5.2 If in (13)-(14) we put $\tilde{a}_{1}=1, \tilde{a}_{2}=1, \tilde{k}=1$, then the solution of problem (13)-(14) has the form:

$u_{1}(y, x)=\frac{\frac{a_{1}}{a_{2}}+k}{2 k} \widetilde{u}_{1}\left(y, \frac{x}{a_{1}}\right)-\frac{\frac{a_{1}}{a_{2}}-k}{2 k} \widetilde{u}_{2}\left(y,-\frac{x}{a_{1}}\right), x<0$

$u_{2}(y, x)=\widetilde{u}_{2}\left(y, \frac{x}{a_{2}}\right), 0<x$

Corollary 5.3 The solution of the boundary value problem (11) is given by similar Poisson's formula:

$$
\begin{aligned}
& u_{2}(y, x)=\frac{y}{\pi} \frac{2 k}{\frac{a_{1}}{a_{2}}+k} \int_{-\infty}^{0} \frac{1}{\left(\frac{x}{a_{2}}-\xi\right)^{2}+y^{2}} f_{1}\left(a_{1} \xi\right) d \xi+ \\
& +\frac{1}{2 \sqrt{\pi t}} \int_{0}^{\infty}\left(\frac{\frac{a_{1}}{a_{2}}-k}{\frac{a_{1}}{a_{2}}+k} \frac{1}{\left(\frac{x}{a_{2}}+\xi\right)^{2}+y^{2}}+\frac{1}{\left(\frac{x}{a_{2}}-\xi\right)^{2}+y^{2}}\right) f_{2}\left(a_{2} \xi\right) d \xi, x>0 \\
& u_{1}(y, x)=\frac{1}{2 \sqrt{\pi t}} \int_{-\infty}^{0}\left(\frac{k-\frac{a_{1}}{a_{2}}}{\frac{a_{1}}{a_{2}}+k} \frac{1}{\left(\frac{x}{a_{1}}+\xi\right)^{2}+y^{2}}+\frac{1}{\left(\frac{x}{a_{1}}-\xi\right)^{2}+y^{2}}\right) f_{1}\left(a_{1} \xi\right) d \xi \\
& +\frac{1}{2 \sqrt{\pi t}} \int_{0}^{\infty} \frac{2 \frac{a_{1}}{a_{2}}}{\frac{a_{1}}{a_{2}}+k} \frac{1}{\left(\frac{x}{a_{1}}-\xi\right)^{2}+y^{2}} f_{2}\left(a_{2} \xi\right) d \xi, \quad x<0 .
\end{aligned}
$$

\section{References}

[1] Marchenko, V. A. (2011), Sturm-Liouville operators and applications (2 ed.), Providence: American Mathematical Society

[2] I. A. Kipriyanov,Lyakhov L.N., Raykhelgauz L.B.Singular Heat Equation with -Bessel Operator. Fundamental Solutions Journal of Mathematical Sciences V. 188, No. 3, 2013. pp. 283-293.

[3] Samko, S.G., Kilbas, A.A. and Marichev, O.I., Fractional Integrals and Derivatives: Theory and Applications, Gordon and Breach, New York (1993).

[4] Carslaw, H. S.; Jaeger, J. C. , Conduction of Heat in Solids (2nd ed.), 1959,Oxford University Press,

[5] Evans, L.C. , Partial Differential Equations, American Mathematical Society,1998.

[6] R. Courant, D. Hilbert, Methods of Mathematical Physics, vol II. Interscience (Wiley) New York, 1962.

[7] Polyanin, A. D. and A. V. Manzhirov, Handbook of Mathematics for Engineers and Scientists, Chapman and Hall/CRC Press, 2007.

[8] E. Mogileva, O. Yaremko, Hermite functions with discontinuous coefficients for the solution of fractal diffusion retrospective problems, International journal of applied mathematics and informatics,Issue 3, Volume 7, 2013, p.78-86. 
[9] O.E. Yaremko,Matrix integral Fourier transforms for problems with discontinuous coefficients and transformation operators, Reports Of Academy Of Sciences, Volume. 417, Issue 3, 2007, p. 323-325.

[10] Bavrin I.I., Matrosov V.L., Jaremko O. E.(2006) Operators of transformation in the analysis, mathematical physics and Pattern recognition. Moscow, Prometheus, p 292.

[11] O. Yaremko, V. Selutin, N. Yaremko, The Fourier Transform with Piecewise Trigonometric Kernels and its Applications, WSEAS transactions on mathematics, Volume 13, 2014, pp. 615-625/

[12] O.E. Yaremko, Transformation operator and boundary value problems, Differential Equation. Vol.40, No. 8, 2004, pp.1149-1160 